

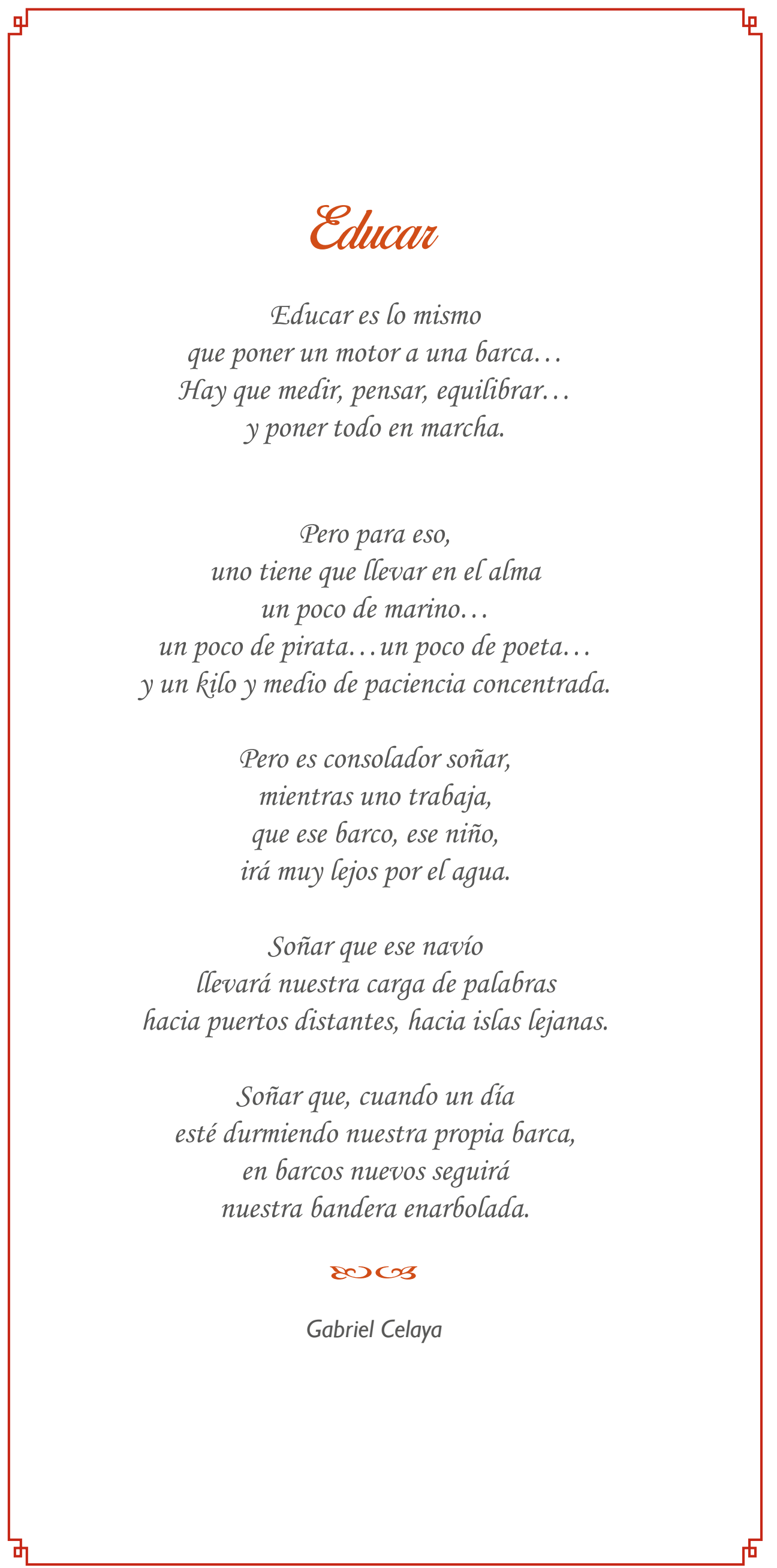




\title{
3. Eficiencia educativa en Honduras, años 2006 y 2011
}

\author{
Martha Lineth González ${ }^{1}$ \\ Recibido 12-06-2017 / Aceptado 17-07-2017
}

\begin{abstract}
RESUMEN: La población en edad escolar es la porción de la población que comprende entre los cinco y los diecisiete años y demanda de forma constante servicios educativos de calidad. Para conocer las demandas de la población en edad escolar es necesario conocer la distribución de la misma, atendiendo a variables como sexo, edad, departamento y área de residencia. Tras ese análisis se puede afirmar que la población en edad escolar se distribuye de forma desigual. La cobertura educativa ha presentado grandes logros para las personas en edades que corresponden a los dos primeros ciclos de la educación básica, evidenciándose un descenso de la cobertura en el tercer ciclo. El nivel de educación pre básica presentó mayores logros de 2006 a 201 I, especialmente por los esfuerzos para la universalización de este nivel. La inversión en educación ha descendido pero este factor no ha impedido logros notables en la cobertura, aunque en algunos departamentos no se justifica los montos invertidos dados los descensos en la cobertura. En vista que las demandas sociales son crecientes y de carácter cambiante y que la población en edad escolar no está exenta de transformaciones; se prevé que para 202 I los niveles educativos que se revestirán de mayor demanda serán el nivel educativo medio y superior, condición que debe considerarse de forma directa en la planificación estatal de la educación nacional.
\end{abstract}

Palabras claves: edad escolar, cobertura, transición demográfica

\begin{abstract}
The school-age population is the portion of the population comprising between five and seventeen years old and constantly demands quality educational services. To meet the demands of the school age population is necessary to examine the distribution of it by gender, age, residence and department, after this analysis we can say that the school-age population is distributed unevenly. Educational coverage has presented great achievements for people, whose ages correspond to the first two cycles of basic education, showing a decrease of coverage in the third cycle. Preschool education level had the most achievements from 2006-20II, due to the efforts for the universalization of this level. Investment in education has decreased, but this factor has not stopped remarkable achievements in coverage; in some departments, the amounts invested do not justify the declines in coverage. Given that social demands are growing and changing and that the school-age population is not expected to have changes it is expected that in 202 I the educational levels with the highest demand will be the middle and higher level of education. This condition must be considered directly in state planning of national education.
\end{abstract}

Keywords: school age, coverage, demographic transition.

\section{Introducción}

La educación es el medio por excelencia para alcanzar el desarrollo de los pueblos, y a su vez una de las líneas de acción más importante en materia de política pública. Es un derecho fundamental de los habitantes de un país y está ampliamente incluida en la agenda legislativa, económica y de planificación estratégica en todas las naciones del mundo, tomando mayor auge desde los años 90's a partir de la Declaración Mundial sobre Educación para Todos de Jomtien.

El goce del derecho de acceso a la educación implica una gran responsabilidad estatal para ser garantizado de forma sostenible. Por ende, la inclusión y la equidad en la distribución de la oferta educativa son temas de gran interés y discusión desde la perspectiva económica, social y demográfica, y requiere de un análisis profundo, que permita al Estado ofrecer servicios educativos oportunos y de calidad; y a los demandantes de los mismos, gozar de los beneficios individuales y colectivos que brinda la educación.
Es innegable que en materia educativa existe tanto oferta como demanda y el delicado equilibrio entre ambas, está mediado por la voluntad política, los objetivos en planificación estatal, la disponibilidad de recursos humanos y financieros con que cuenta el Estado, así como la distribución y estructura etaria de la población demandante de la misma.

Éste último elemento es determinante de las fallas y desequilibrios entre la oferta y demanda en tema de servicios educativos pues el país ha experimentado vertiginosos cambios en los últimos años, tanto en estructura por sexo y edad de la población, así como en la distribución geográfica de la misma.

La necesidad de analizar la demanda y oferta educativa desde un enfoque demográfico es imperante pues permite identificar las relaciones estrechas entre población y prestación de servicios educativos. Partiendo de la premisa que la educación es el medio y fin del desarrollo de los individuos tanto en carácter personal como social, y debe ser prioridad pública. En tal sentido, cualquier acción estatal debe responder de forma acertada a la realidad.

\footnotetext{
${ }^{1}$ Máster en Demografía y Desarrollo UNAH, Lic. en Ciencias Sociales UPNFM, correo electrónico marthalin2005 @yahoo.com
} 


\section{Metodología}

En este estudio se intenta demostrar la respuesta del sistema educativo a la dinámica demográfica de la población en edad escolar para Honduras ${ }^{2}$ en los años 2006 y 2011. Para tal efecto se hizo uso de la Encuesta Permanente de Hogares (EPHPM) de los años seleccionados. Es una investigación con un enfoque cuantitativo y diseño no experimental (Hernández, Fernández \& Baptista: 2008) dado que las características de la población y muestra seleccionada no han sido manipuladas durante los años seleccionados para tal estudio. Además del diseño antes mencionado el estudio es de carácter longitudinal de tendencia, puesto que analiza los cambios en la población en edad escolar en los años 2006 y 2011.

Se seleccionó como objeto de estudio la población cuya edad cumplida oscila entre los 5 y 19 años de edad. Además, se extendió la edad escolar en dos años adicionales en relación al marco legal nacional para no excluir a los individuos que presentan extra edad, repitencia o rezago en su respectivo nivel educativo. La extra edad no es una variable incluida para el análisis exhaustivo en el presente estudio.

Es necesario aclarar que los departamentos de Gracias a Dios e Islas de la Bahía no cuentan con representatividad dentro de la EPHPM 2011. Esta determinación es responsabilidad del Instituto Nacional de Estadísticas (INE) e independiente a este estudio.

\section{Discusión de resultados}

\subsection{Población hondureña en edad escolar, 2006-2011}

La transición demográfica es el enfoque teórico que sustenta los hallazgos de esta investigación, por lo cual en este capítulo se demostrará la evolución de las cohortes en edad escolar, que se están ajustando a un descenso de la natalidad y un aumento sostenido de la población joven, etapa que se conoce como bono demográfico y que según Manuel Flores (2007) en Honduras se está perfilando para el mayor auge en las próximas décadas, específicamente de 2015 a 2040.

La evolución de la población en el marco de la transición demográfica se convierte en un reto para la creación de oportunidades laborales, que a su vez descansan en las oportunidades educativas. En ese contexto los siguientes datos, gráficos y mapas, muestran la evolución de la población en edad escolar en dos momentos históricos, 2006 y 2011 y una proyección de la población en edad escolar mediante una función lineal de crecimiento, cuyos indicadores de análisis son especialmente edad y sexo y permiten establecer los cambios operados en la población en edad escolar.

\section{$\rightarrow$ Población en edad escolar según sexo}

Entre los años 2006 y 2011, se observa un descenso de la población en edad escolar en el caso del sexo femenino, tal descenso no es tan marcado, ya que representa un $1.3 \%$ y en contraparte un aumento de la población masculina en edad escolar que significó un $1.56 \%$.

La población en edad escolar aumentó en 56,849 individuos, lo que representa un aumento en la demanda potencial en educación, cantidad que utilizando una función lineal de crecimiento de la población representa 11,430 individuos promedio por año entre 2006 y 2011, y permite proyectar una población total en edad escolar de 3, 124,064 individuos para 2021. Esto demuestra un aumento constante en el porcentaje de varones en edad escolar con un $2.3 \%$ de aumento y acentúa el descenso de la población en edad escolar con sexo femenino con un $2.3 \%$ de decrecimiento (Gráfico $N^{\circ} 1$ )

\section{Gráfico $N^{\circ} 1$. Honduras: distribución porcentual de la población en edad escolar según sexo. 2006, 2011 y 2021}

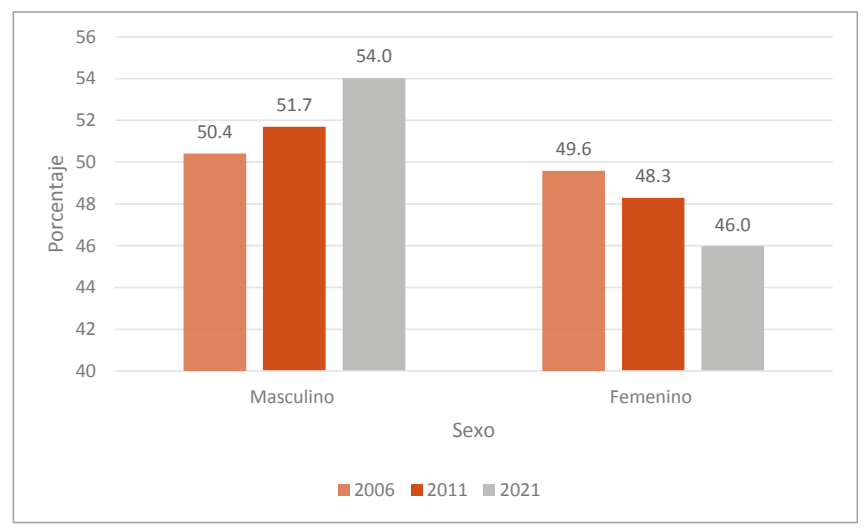

Fuente: Elaboración propia con base en la EPHPM. 2006-20II

\section{Población en edad escolar según edad simple}

La edad simple es el número de años cumplidos de cada individuo en el momento de aplicación del instrumento, este criterio además coincide con la estructura del sistema educativo nacional que agrupa a los alumnos en grados escolares con un año de edad simple como referencia. Desde primer grado con una edad de referencia de seis

\footnotetext{
${ }^{2}$ En correspondencia con el marco legal de Honduras, la población en edad escolar abarca desde los 5 años de edad hasta los 17 años en los niveles de educación Pre Básica, Básica y Media.
} 
años hasta segundo de año de bachillerato con diecisiete años como edad de referencia.

En el año 2006, la edad de la población escolar presentaba una fuerte concentración de individuos en las edades simples $8,10,12$; con $7.5 \%, 7.33 \%$ y $7.2 \%$ respectivamente, evidenciando un leve descenso de la población con mayor edad, siendo la edad de 19 años la que menor frecuencia presentó, apenas un $4.89 \%$, tanto para el sexo femenino como masculino. No se observa predominio de algún sexo pues las fluctuaciones de una edad a otra son muy leves.

Para el año 2011, las edades simples que mayor porcentaje presentaron fueron los 11, 14 y 15 años, con un $7.60 \%, 7.49 \%$ y $7.28 \%$ respectivamente. Se evidencia un traslado de la población en edad escolar a la cohorte superior, cambio de la estructura que demuestra que la transición demográfica en Honduras es cada vez más marcada.

Entre 2006 y 2011 la estructura de la población experimentó variaciones considerables en las primeras edades simples, donde el descenso, se hace más notorio con un crecimiento negativo tanto de la población femenina y masculina. Existe una tendencia a la baja de la población en edad escolar por los cambios en la natalidad y fecundidad ligados al proceso de transición demográfica en nuestro país. Hecho que se constata con el drástico descenso de los individuos en edad escolar desde los 6 hasta los 15 años.

Entre 2006 y 2021, se proyecta un crecimiento negativo de la población en edad escolar distribuidos desde los 6 años en un $4.7 \%$. Para los 7 años un $3.66 \%$. Para los 8 años de edad un $5.12 \%$. En contraparte se muestra un traslado hacia las edades inmediatas superiores de la población escolar de un año de estudio a otro. Debido al hecho natural del crecimiento de los individuos y su posterior traslado a otras edades, entre 2006 y 2021 , varió a los 15 años en un $2.38 \%$ de aumento, lo mismo que para los 16 años en un $0.87 \%$; para los 17 años se constató un $3.09 \%$ siendo evidente el traslado de la población en edad escolar a otro grupo etario y en el caso de los 19 años un crecimiento de la población en edad escolar de $2.36 \%$ (Gráfico № 2 y №3).

Todas estas transformaciones en la estructura de la población se traducen en demandas educativas que deben enfatizar en la preparación de la infraestructura, número de maestros, montos de inversión que respondan al traslado de la población en edad escolar de los grados escolares del I ciclo del nivel de educación básica a nivel de educación media.

Es posible afirmar que los efectos de la transición demográfica en nuestro país están generando un descenso de la población en edad escolar acompañado de un traslado de dicha población de un nivel educativo a otro. Ese traslado se da de forma simultánea en la concentración de demandas de servicios educativos del nivel básico al nivel de educación media y superior.

Gráfico $N^{\circ} 2$. Honduras: distribución porcentual de la población masculina en edad escolar según edad simple, 2006, 2011 y 2021

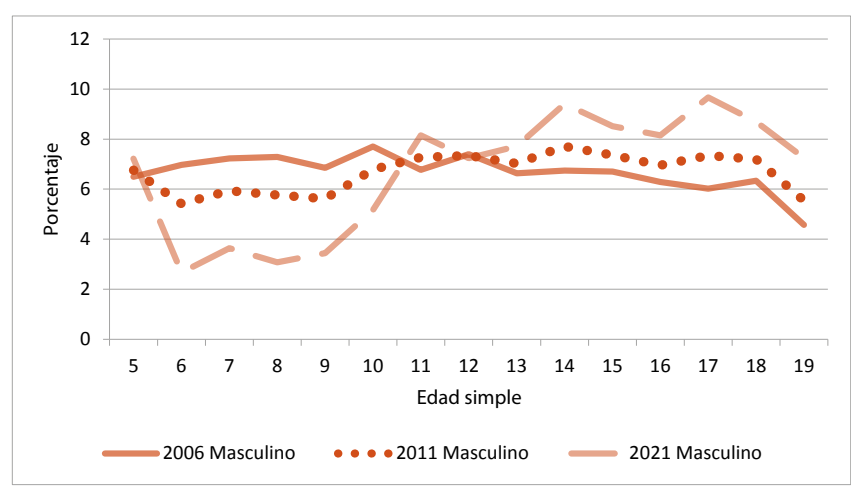

Fuente: Elaboración propia con base en la EPHPM. 2006-20II.

Gráfico $N^{\circ} 3$. Honduras: distribución porcentual de la población femenina en edad escolar según edad simple, 2006, 2011 y 2021

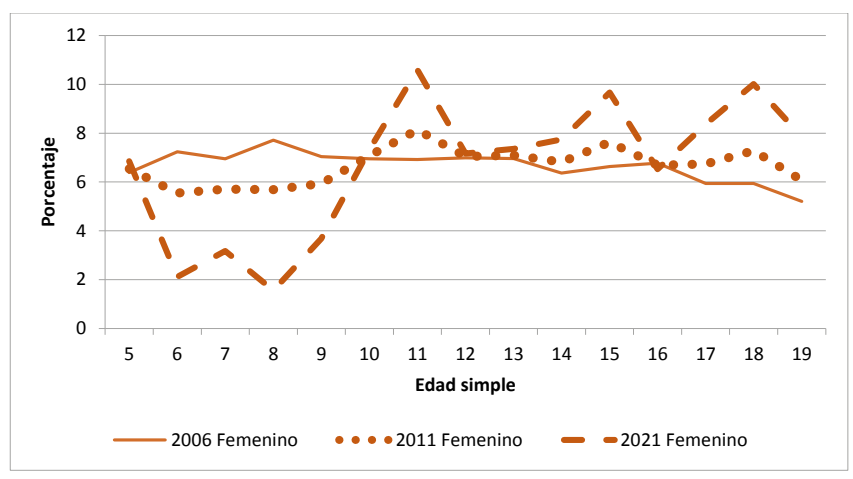

Fuente: Elaboración propia con base en la EPHPM. 2006-20II.

\section{$\longrightarrow$ Distribución de la proyección de población en edad escolar según departamento}

La proyección de la población es una herramienta indispensable en la planificación estatal especialmente las relacionadas a demandas sociales que deben responder a las variaciones de la población. En este sentido, los cambios que se desarrollaran en la distribución de la población en edad escolar para los próximos 10 años deben tomarse en cuenta para la toma de decisiones acertadas. Basados en la proyección de población en edad escolar 
realizada en este estudio, se puede afirmar que para la población masculina los departamentos con porcentajes más altos serán Francisco Morazán, Cortés, Yoro y Olancho, cada uno con un $18.52 \%, 18.14 \%, 7.07 \%$ y $6.88 \%$ respectivamente. En contraste los departamentos de Ocotepeque se proyecta con $1.66 \%$, Valle, $2.19 \%$ y La Paz con $2.33 \%$ son los que presentarán la menor concentración (Mapas $\mathrm{N}^{\circ} 1, \mathrm{~N}^{\circ} 2$ y $\mathrm{N}^{\circ} 3$ )

En términos generales, la población masculina en edad escolar presenta un leve descenso porcentual para 2011 y un repunte a 2021, estas variaciones son muy leves y únicamente los departamentos de Olancho y Santa Bárbara sufrirán un descenso de su población masculina en edad escolar para 2021.

La población femenina en edad escolar para 2021, se encontrará concentrada en los departamentos de Francisco Morazán (19.5\%), Cortés (18.59\%), Yoro (7.48\%) y Olancho (6.43\%). Por el contrario, los departamentos de Ocotepeque, La Paz y Valle, mostrarán 1.7\%, 2.18\% y $2.32 \%$; por lo tanto, serán los que concentrarán menor población.

La población femenina en edad escolar para 2021, muestra un descenso generalizado en relación a 2011, ya que sólo cinco (5) departamentos presentarán un aumento en puntos porcentuales. Dichos departamentos serán Francisco Morazán, Cortés, Choluteca, Ocotepeque y El Paraíso; cada uno con un aumento de 3.44, 0.96, 0.77, 0.13 y 0.09 respectivamente.

Francisco Morazán presenta un panorama singular siendo que su aumento de población femenina en edad escolar es alto, condición que debe considerarse pues la población femenina de 2006 a 2021, aumentó el porcentaje de cobertura, lo que podría incidir en los porcentajes cobertura para 2021 (Ver apartado 5.3.4. sobre cobertura educativa) lo que requerirá mayor atención gubernamental para este segmento de la población.

Dentro del panorama nacional la población en edad escolar para el año 2021, se encontrará de forma mayoritaria en los departamentos de Francisco Morazán (19.05), Cortés (10.38), Yoro (7.29) y Olancho (6.56); y los de menor concentración serán Intibucá con un $2.41 \%$; Valle, $2.26 \%$; La Paz, $2.25 \%$ y Ocotepeque con un $1.68 \%$.

La dinámica que la población en edad escolar mantiene de 2006 a 2011, refleja un aumento en la mayoría de los departamentos, pero de 2011 a 2021, este aumento sólo es observable en Atlántida, Cortés y Francisco Morazán. Nuevamente Francisco Morazán y Cortés presentan ca- racterísticas muy especiales en relación al resto nacional, ya que de 2011 a 2021 crecerán en 1 y 4.04 puntos porcentuales respectivamente, lo que se traduce en una mayor presión hacia el sistema educativo en estos dos departamentos.

El departamento de Atlántida, también muestra crecimiento, pero éste es de apenas 0.04 puntos porcentuales. Los trece departamentos restantes presentan descensos en el porcentaje de población en edad escolar, este descenso puede estar ligado a la disminución de la natalidad que se ha venido presentando en el país o a procesos migratorios internos, especialmente al evidenciarse el aumento porcentual en Francisco Morazán y Cortés.

En cualquiera de las vías que se oriente el fortalecimiento del sistema educativo se debe considerar a la población en edad escolar como el mayor determinante, en otras palabras si se toma la decisión de fortalecer a los departamentos de Francisco Morazán y Cortés las presiones sobre éstos serán un detonante, ya que puede crearse en la población en general la concepción que sólo al migrar los niños y jóvenes pueden acceder a servicios educativos de calidad y en medio de este proceso existirá inevitablemente una fracción de la población en edad escolar que no podrá sufragar los costos del mismo.

\subsection{Demanda de la educación en los niveles de Pre Básica, Básica y Media}

Cabe aclarar que la demanda potencial de educación incluye la totalidad de la población en edad escolar y que de ésta; el porcentaje de población en edad escolar que asiste a un centro educativo se convierte en la demanda real de educación.

La cobertura en educación se entiende como la demanda educativa, la cual es el porcentaje de la población en edad escolar que está inserto en algún nivel educativo, ya sea este el correspondiente a su edad de referencia legal para cada nivel o cualquier otra edad, ya sea resultado de la extra edad o repitencia.

La cobertura educativa es desigual en términos de sexo, edad, nivel educativo y área de residencia, este apartado presentará un análisis de cada uno de los elementos diferenciales de la cobertura escolar.

\section{Cobertura educativa según sexo}

Para el año 2006, la cobertura en edad escolar era de $50.42 \%$ para los individuos de sexo masculino y un $49.58 \%$ para el sexo femenino. Evidenciando apenas un $0.84 \%$ de superioridad masculina en la conformación de 
la población en edad escolar que asiste a un centro educativo.

Para 2011, la distribución de la cobertura educativa según sexo era de un $51.67 \%$ de los varones y un $48.33 \%$ de las niñas y jóvenes del sexo femenino. En este año se modificó la superioridad masculina sobre la femenina ya que la cobertura educativa descendió para el sexo femenino en un $1.25 \%$.

En la composición de la población dentro de la cobertura educativa, cabe resaltar que la población excluida de tal cobertura en ambos años ha presentado un predominio del sexo masculino sobre el femenino, lo que indica que los varones son más susceptibles ante los factores que propician la exclusión del sistema educativo tales como el trabajo infantil, la paternidad adolescente, la migración entre otros.

Un 53.22\% de la población excluida del sistema educativo eran varones en 2006, para 2011 este porcentaje represento el $55.94 \%$; tendencia que además es visible y con un claro crecimiento de $2.72 \%$ de la población masculina excluida del sistema educativo.

\section{- Cobertura educativa según edad simple}

La edad es un determinante de la cobertura educativa, ya que con el crecimiento de los individuos en edad escolar-

\section{Mapa $\mathrm{N}^{\circ}$ 1. Honduras: distribución porcentual de la población en edad escolar según departamento, 2006-2011}

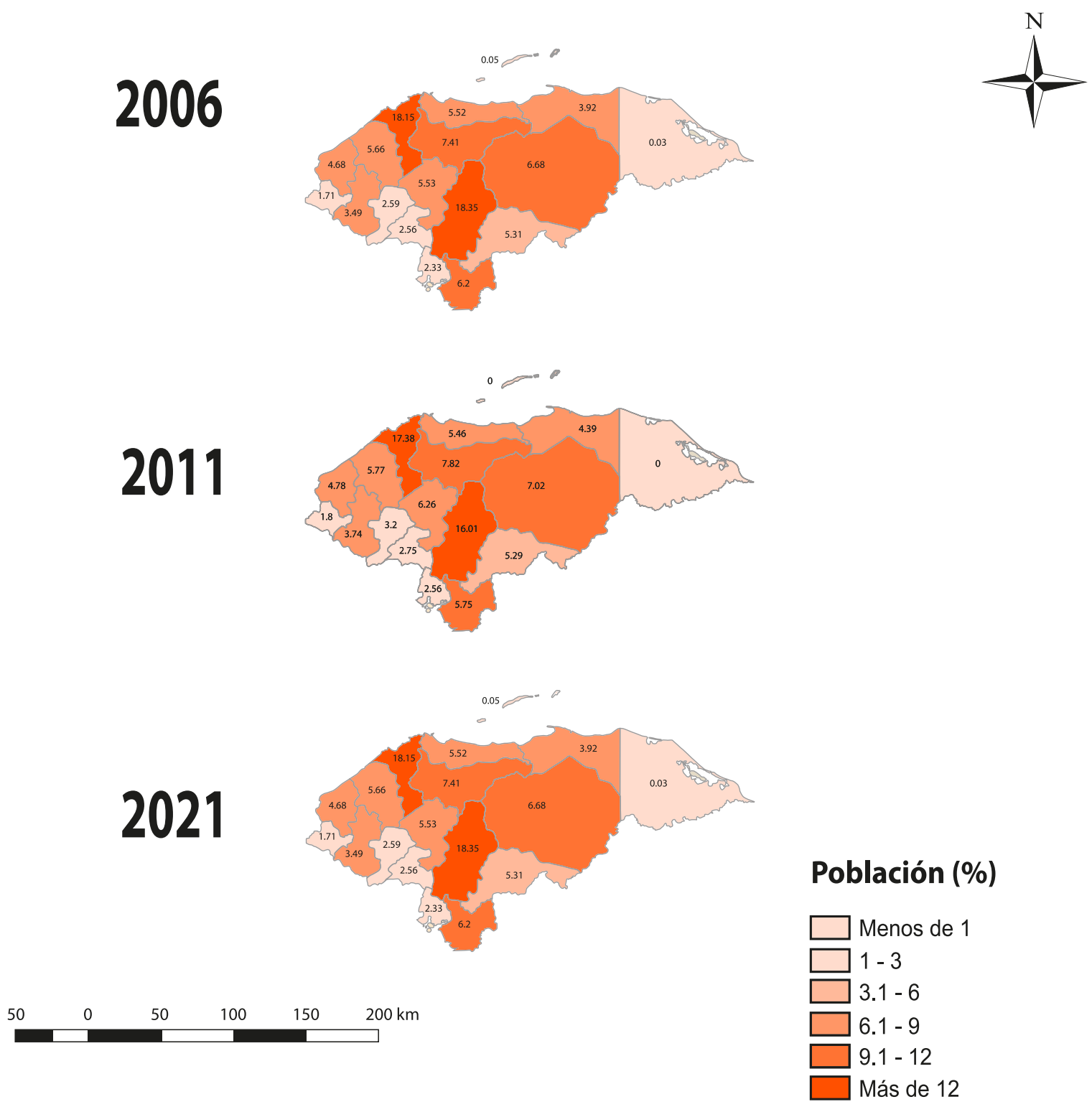


Gráfico $N^{\circ}$ 4. Honduras: distribución porcentual de la población en edad escolar según asistencia un centro educativo y sexo, 2006-2011

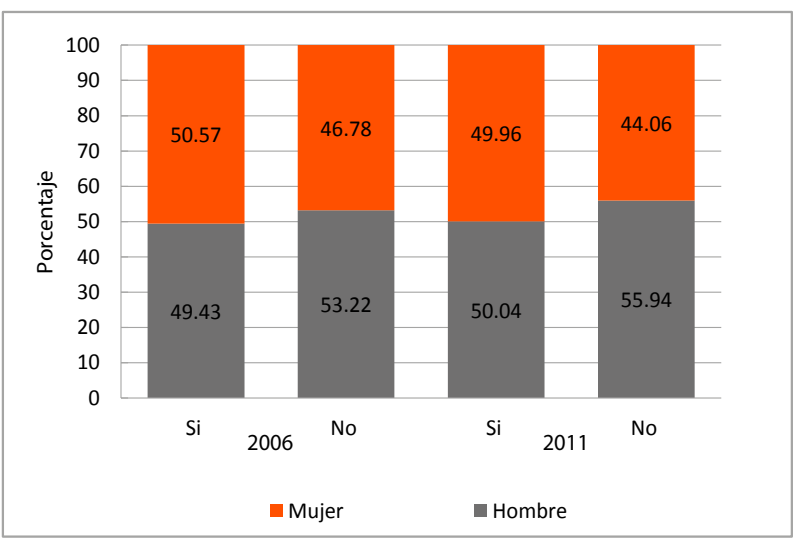

Fuente: Elaboración propia con base en la EPHPM. 2006-20II.

se enfrentan a condiciones socioeconómicas, culturales y familiares que actúan como elementos excluyentes del sistema educativo, entre estos la necesidad de sustentar el hogar, la inexistencia de centros educativos en las áreas cercanas, la necesidad de migrar internamente para continuar sus estudios, la maternidad o paternidad adolescente, entre otros factores.

Para establecer el comportamiento de la cobertura educativa según la edad simple es necesario aclarar que esta es desigual según la estructura etaria de la población demandante y va en descenso directamente proporcional al aumento de la edad.

Para 2006, el comportamiento de la cobertura educativa es irregular ya que para los 5 años de edad es fue de $61.96 \%$, porcentaje que gradualmente va en aumento hasta llegar a los 9 años de edad en los que la cobertura es realmente importante con un $96.15 \%$, pero este porcentaje empieza a descender al punto que a los 15 años de edad se ha reducido hasta un $60.18 \%$ y sigue con un descenso dramático; a los 19 años con un $30.90 \%$, como se muestra en el Gráfico № 5.

En 2011, la cobertura educativa se presentó para los mismos años de edad, es decir, 5, 9, 15 y 19 años, con $70.37 \%, 94.29 \%$, 59.46\%, y $32.43 \%$, respectivamente. Un cambio notable entre 2006 y 2011 muestra que la edad que mayor cobertura presentó varío de los 9 a los 10 años con un $96.72 \%$ para 2011.

En cada una de las edades simples la cobertura aumentó evidenciando un sistema educativo nacional más incluyente, cabe resaltar que el aumento más significativo se presentó en las edades de 5 y 6 años que aumentaron de en un 8.41 y un 7.37 puntos porcentuales respecti- vamente. Este aumento está ligado a la obligatoriedad que tiene la educación formal en esas dos edades desde la aprobación de la Ley Fundamental de Educación en 2011.

A partir de los 7 años de edad hasta los 12 años la cobertura también aumentó, pero los cambios fueron menos marcados, pues oscilan entre el $0.20 \%$ y $0.40 \%$ de un año a otro. Se debe recalcar que estas edades coinciden con la Educación Básica en sus primeros dos ciclos en los cuales se han enfocado grandes esfuerzos estatales, como una de las metas de los Objetivos del Milenio (ODM) y el Plan de Nación, Visión de País.

Es probable que los elementos que determinan la cobertura en el nivel de Educación Básica en sus primeros dos ciclos, sea difícil de cambiar, y alcanzar un aumento porcentual de la cobertura se consiga lentamente; ya que al ser superior al 93\% requerirá esfuerzos estatales aún más altos para lograr un aumento.

Gráfico $N^{\circ}$ 5. Honduras: distribución de porcentual de la cobertura educativa según edad simple, 2006 - 2011

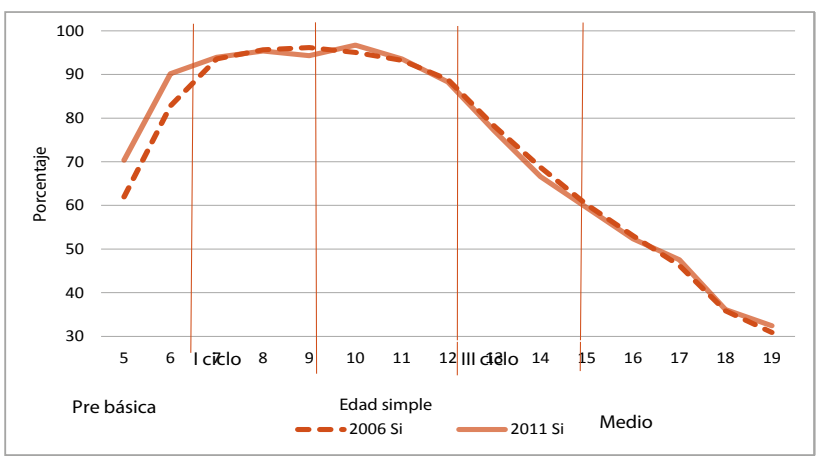

Fuente: Elaboración propia con base en la EPHPM. 2006-20II.

En contraparte las edades que van de los 13 a los 19 años presentaron un comportamiento irregular pues de un año a otro se evidencian descensos y aumentos en distintas edades. Tal es el caso de la cobertura educativa a los 13,14 , y 15 años la cual descendió 1.03; 2.15; y un 0.72 puntos porcentuales de 2006 a 2011. En contraste con la cobertura a los 17, 18 y 19 años de edad, que presenta un aumento de un 1.3; 0.23; y 1.53 , respectivamente, lo que demuestra que la cobertura educativa no solo desciende drásticamente de los 12 a los 13 años de edad, sino que desciende de un año de edad a otro, lo cual permite afirmar que los esfuerzos para elevar la cobertura educativa que ya han alcanzado aumento constante en las edades de 5 a 12 años deben orientarse también a las edades de 13 a 15 años. Especialmente bajo el criterio que, a partir de 2011 con el cambio en el marco legal de la educación nacional, también es obligatorio el III ciclo de Educa- 
ción Básica que comprende las edades de 13 a 15 años (Gráfico $N^{\circ} 5$ ).

Se puede afirmar que los ciclos del nivel de educación básica tienen una cobertura desigual, muy alta entre los siete y 11 años que comprenden el I y II ciclo, pero drásticamente baja en las edades de 13 a 15 años que comprende el III ciclo, dicho análisis se profundizará en el siguiente apartado.

\subsection{Inversión total e inversión en infraestructura educativa por alumno según departamento, $2006-2011$}

Entre los años 2006 y 2011, solamente los programas y erogaciones que se mantuvieron constantes fueron los pagos de sueldos y salarios docentes y la inversión en infraestructura educativa. Los indicadores fundamentales analizados en este apartado son:

\section{Inversión total por alumno}

Cada uno de los montos permite calcular, la inversión total por alumno, en ambos años. Se define como el cociente entre el monto invertido y el número de individuos que están insertos en el sistema educativo de cada año.

Los montos de inversión se han deflactado para corregir el efecto de la inflación de cada año utilizando como base el año 1999.

La inversión total por alumno es un indicador que expresa cuantos Lempiras invirtió el Estado en cada individuo en edad escolar que estuvo inserto en el sistema educativo, este indicador incluye cada uno de los programas y responsabilidades financieras que el Estado asume para mantener la oferta educativa. Siendo que, en 2006, la inversión total por alumno fue de Lps 4,070.00 y en 2011, de Lps. 3,079.00. Es evidente el descenso de la inversión total por alumno de un año a otro, puesto que desaparecieron la mayoría de los programas que existían en 2006 , pasando de 13 programas a 7 , de un año a otro.

No es uno de los objetivos de este estudio determinar las causas de la reducción de la inversión total por alumno, solamente establecer la relación que se presenta entre la reducción de la inversión total y el descenso de la cobertura educativa, ya que el promedio nacional en cobertura para 2006 era de $71.27 \%$ y descendió a $59.39 \%$.

La reducción en la inversión educativa no es el único elemento explicativo del descenso de la cobertura, pero por su relación directamente proporcional puede tratarse de un determinante de gran relevancia.
Gráfico $N^{\circ}$ 6. Honduras: distribución de la inversión total por alumno, 2006-2011

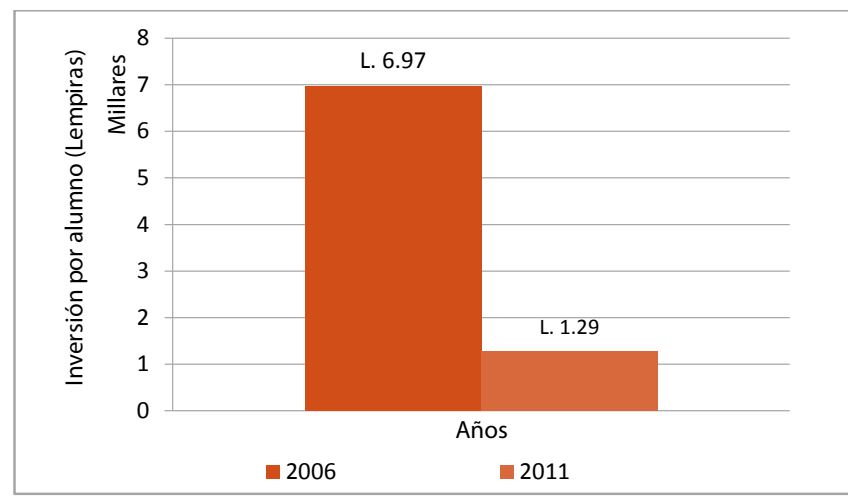

Fuente: Elaboración propia con base presupuesto nacional aprobado. 201 I. SEFIN y EPHPM 2006 y 2011

\section{Inversión en infraestructura educativa}

Para 2006 los departamentos con más alto índice de inversión en infraestructura por alumno fueron Ocotepeque y Francisco Morazán con Lps. 23.85 y Lps. 20.04 anualmente. La inversión por alumno en cada departamento es claramente desigual, ya va desde los Lps. 0.00 por alumnos en el caso de La Paz y Yoro, hasta la altísima inversión de Ocotepeque y Francisco Morazán.

Además de ser muy variable; puesto que los criterios de priorización obedecen a programación del sistema educativo más que a las demandas de la población en edad escolar, ya que los departamentos con mayor cobertura y más altos porcentaje de población de población en edad escolar no corresponden directamente a los de más alta inversión en infraestructura.

Para 2011, la inversión en infraestructura educativa desciende considerablemente ya que a precios constantes pasa de Lps. 16, 228,838.00 millones a Lps. 15, 000,000.00. En promedio pasa de Lps. 6.04 invertidos a Lps. 2.70 anualmente.

Este descenso evidente de la inversión por alumno coincide con un descenso de la cobertura escolar de un año a otro. Además, se puede afirmar que la inversión por alumno sólo aumentó en el departamento de Colón, y en los 17 departamentos restantes se redujo drásticamente. Al igual que el promedio nacional.

Para 2006 solo Yoro, La Paz, Atlántida y Colón presentaban una inversión en infraestructura inferior a los Lps. 3.00 por alumno anualmente, categoría que aumentó claramente en 2011, donde; Atlántida, Comayagua, Copán, Cortés, Choluteca, El Paraíso, Intibucá, La Paz, Lempira, Olancho, Santa Bárbara, Valle y Yoro; se sumaron (Mapa № 2)

Revista Población y Desarrollo: argonautas y caminantes 


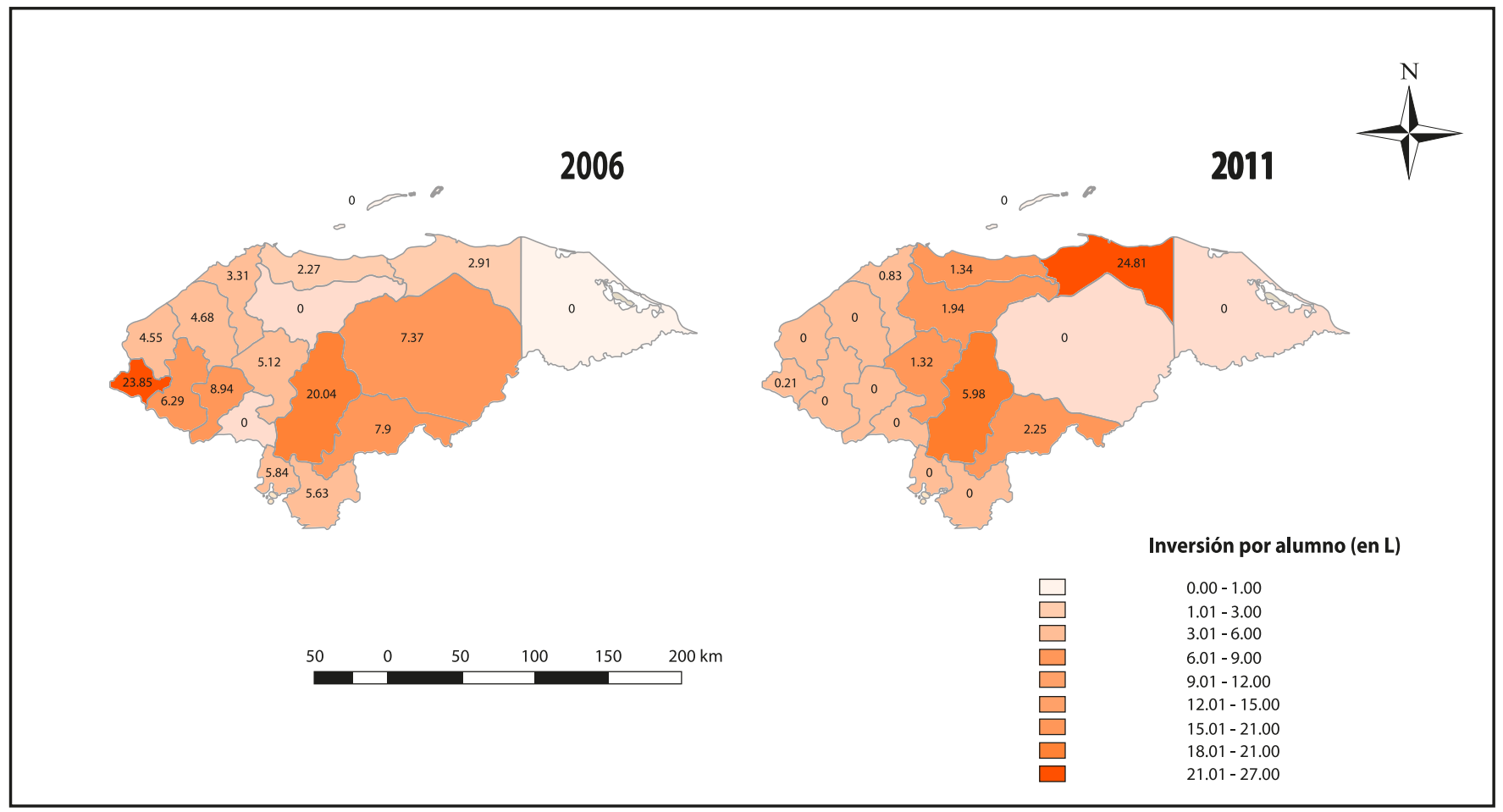

Fuente: Elaboración propia en base a EPHPM 2006 - 20II SEFIN Unidad de Inversión Pública; Presupuesto 2006 - 20 II SINIT Honduras

En términos generales la inversión en infraestructura educativa ha descendido de forma muy marcada, pero por sí sola no explica los cambios en la cobertura educativa, puede establecerse una relación entre ambos indicadores, pero la desvinculación de las fuentes de datos demográficos y financieros limita profundamente el análisis.

No se debe menospreciar el hecho que la inversión en montos totales creció de 2006 a 2011, pero que los programas que perciben la mayor cantidad de fondos no han podido mantener mucho menos elevar la cobertura educativa a nivel nacional.

\section{4 Î́ndice de eficiencia educativa}

El índice de eficiencia educativa es un instrumento que permite valorar la influencia de diversos factores relacionados al equilibrio entre demanda y oferta educativa. Entre ellos el porcentaje de cobertura educativa, la relación alumno/docente y la inversión por alumno.

Cada uno de los indicadores antes señalados tiene una relación directa con la calidad educativa y no solo en esta sino también en la eficiencia, esta última se ha cuantificado tradicionalmente con indicadores como la cobertura educativa, la evaluación docente y los métodos y técnicas didácticas.
Pero la eficiencia es un tema de amplio debate, ya que ante la necesidad de priorizar el destino de los recursos con los que cuentan los Estados, no se trata únicamente de asignarlos y velar por su uso adecuado; también se trata de redistribuirlos según los resultados de los programas, proyectos, sectores e iniciativas estatales que más beneficios brinden a la sociedad.

\section{Cuadro $N^{\circ} 1$. Honduras: índice de eficiencia educativa según nivel educativo, 2006- 2011}

\begin{tabular}{|c|c|c|c|c|c|c|c|c|c|}
\hline \multirow[b]{2}{*}{ 哫 } & \multirow[b]{2}{*}{ 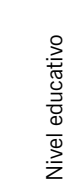 } & \multicolumn{3}{|c|}{ Indicadores } & \multicolumn{3}{|c|}{ Estimación } & \multirow[b]{2}{*}{.0. } & \multirow[b]{2}{*}{ 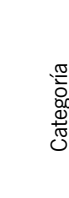 } \\
\hline & & 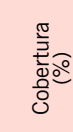 & 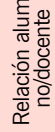 & 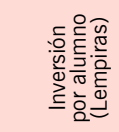 & $\begin{array}{l}\frac{\pi}{3} \\
\frac{t}{0} \\
\frac{0}{0} \\
ن\end{array}$ & 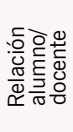 & 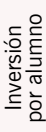 & & \\
\hline \multirow{3}{*}{2006} & $\begin{array}{l}\text { Pre } \\
\text { básico }\end{array}$ & 72.24 & 44 & $6,972.00$ & 3 & 4 & 3 & 10.00 & Alta \\
\hline & $\begin{array}{l}\text { Bási- } \\
\text { co }\end{array}$ & 74.52 & 37 & $6,972.00$ & 3 & 4 & 3 & 10.00 & Alta \\
\hline & Medio & 34.72 & 18 & $6,972.00$ & 2 & 2 & 3 & 7.00 & Media \\
\hline \multirow{3}{*}{2011} & $\begin{array}{l}\text { Pre } \\
\text { básico }\end{array}$ & 78.43 & 27 & $1,287.00$ & 4 & 3 & 1 & 8.00 & Media \\
\hline & $\begin{array}{l}\text { Bási- } \\
\text { co }\end{array}$ & 73.60 & 28 & $1,287.00$ & 3 & 3 & 1 & 7.00 & Media \\
\hline & Medio & 36.92 & 10 & $1,287.00$ & 2 & 1 & 1 & 4.00 & Media \\
\hline
\end{tabular}

Fuente: Elaboración propia con base en EPHPM, SEE Y SIAFI

${ }^{3}$ Calculado a precios constantes en Lempiras. Contabilizado como fuente de financiamiento del tesoro nacional. 
Si bien es cierto que la educación debe ser prioridad nacional, por eso con mayor razón las decisiones deben ser las más acertadas, dado que elementos fundamentales como la asignación de personal docente, la distribución de la inversión y los cambios en población demandante de servicios educativos, pueden aumentar o disminuir la eficiencia.

De 2006 a 2011, la población en edad escolar ha cambiado en todo el país, la inversión ha disminuido, la relación alumno/docente es desigual; y la relación alumno/ centro ha experimentado cambios significativos; así como el marco legal que regula la educación nacional pasó de la Ley Orgánica de Educación a la Ley Fundamental, estos hechos de gran relevancia se han valorado para establecer un índice de eficiencia educativa, cuyos resultados son consecuencia de la valoración de cada indicador.

De esta manera se puede afirmar que los logros en educación son prometedores en cuanto a la cobertura educativa evidenciando un aumento en cada nivel, este indicador es altamente favorecedor dentro del índice de eficiencia educativa, no obstante, otros elementos se han considerado como poco eficientes, entre ellos la relación alumno/docente, la inversión por alumno. Ya que estos demuestran claramente que son desiguales y que, aunque la cobertura ha aumentado con las adecuaciones correspondientes, en la asignación del personal docente estos avances podrían ser mayores.

Por otra parte, la inversión total por alumno se ha reducido de un año a otro, elemento que por sí mismo no es negativo pero que considerando las demandas educativas crecientes de acuerdo a los cambios en la población son un factor inconsecuente con las transformaciones que la transición demográfica conlleva. Y es además inconsistente con la intención de la universalización de la educación básica, siendo que no se entiende que una menor inversión implica que la labor educativa se realiza en condiciones iguales o de menor calidad en cuanto a instalaciones y logística refiere.

De ahí que la incidencia de la relación alumno/docente y la inversión educativa en la eficiencia educativa haya reducido ésta última, especialmente en el nivel de Educación Media en ambos años de estudio, donde la eficiencia se consideró media, siendo el nivel con menor cobertura y más baja relación alumno/docente. En contraste, en los niveles de educación Pre Básica y Básica se pasó de alta a media (Cuadro $\mathrm{N}^{\circ} 1$ ).

Afirmándose entonces que entre 2006 y 2011, la eficiencia educativa se redujo de predominantemente alta a media.

\section{Conclusiones}

La población en edad escolar se encuentra distribuida de forma desigual. Los departamentos del país con mayor concentración fueron Francisco Morazán y Cortés en ambos años de estudio. Pero se observan cambios significativos en departamentos como Yoro, Atlántida, Colón, Intibucá y Lempira.

- La población en edad escolar ha experimentado un traslado de una edad a otra como consecuencia del crecimiento natural de los individuos, de igual manera este cambio se ve reflejado en la demanda potencial de educación que se va transformando simultáneamente con la población. Esto implicará que el sistema educativo deberá responder de forma eficaz trasladando de un ciclo a otro de la educación básica los recursos necesarios para responder a la demanda.

- La distribución de la población en edad escolar según el nivel educativo es desigual y está concentrada en el nivel de educación básica, este fenómeno es atribuible a que en este nivel se agrupan nueve años de escolaridad y apenas tres años y solamente uno para los niveles de educación media y pre básico respectivamente.

- La cobertura educativa o en otras palabras la población en edad escolar que está inserta en el sistema educativo demuestra a lo largo y ancho del país es desigual. Y la población masculina en edad escolar es la más susceptible de exclusión del sistema educativo en ambos años de estudio, puede ser que la población masculina tenga que afrontar situaciones como el sostenimiento del hogar, influencia de la violencia y grupos delictivos, paternidad adolescente, entre otros, pero se recomienda hacer estudios posteriores que analicen de forma puntal las razones de la exclusión escolar según el sexo.

La cobertura escolar ha descendido en el país de 2006 a 2011. Este descenso es generalizado tanto en el análisis por nivel educativo en la educación básica, por departamento y especialmente por dominio de residencia, donde la cobertura para el área rural ya era baja. En cuanto al nivel de educación pre básica los logros son notables dado los esfuerzos gubernamentales para la universalización del mismo.

La oferta educativa que ofrece el Estado de Honduras, expresada en la inversión en infraestructura educativa por alumno, descendió estrepitosamente de un año a otro. Además, existe una divergencia en el aumento de la inversión en diversos programas de apoyo a la educación y descenso en la cobertura educativa. Tales esfuerzos no están alcanzando logros favorables a la educación nacional al contrastar la inversión educativa y los índices de cobertura. Lo que vuelve imperante una revisión de las formas y 
programas de la inversión en educación focalizando en las necesidades estrictas de la población en edad escolar su distribución según departamento, edad, nivel educativo y sexo. Así como los determinantes que condicionan su inserción al sistema educativo.

- La eficiencia del sistema educativo se redujo de 2006 a 2011, este indicador valoró la condición desfavorable como la relación alumno/centro y la variación de secciones en cada centro, así como la relación alumno/docente; a combinación de estos indicadores demuestra que aunque se han presentado logros favorables en cuanto a la cobertura estos logros serían más altos si existiera una adecuada distribución de los centros educativos y maestros contratados.

\section{Bibliografía}

A Acuerdo Ejecutivo No. 262-2011. Ley Fundamental de Educación. Diario Oficial La Gaceta No. 32,754 Tegucigalpa. Miércoles 22 de febrero de 2012.

Banco Central de Honduras (BCH) Índice de precios al consumidor. Diciembre 2006. Retrieved from http://www. bch.hn/download/ipc_historico/ipc122006.pdf

Banco Central de Honduras (BCH) Índice de precios al consumidor. Diciembre 2011. Retrieved from http://www. bch.hn/download/ipc_historico/2011/ipc122011.pdf

Comisión Económica para América Latina y el Caribe (CEPAL). Comercio Internacional e Integración: Deflactación. Retrieved from http://www.cepal.org/cgi-bin/ getprod.asp?xml=/comercio/noticias/paginas/5/34395/ P34395.xml\&xsl=/comercio/tpl/p18f.xsl\&base=/comercio/tpl/top-bottom.xsl

Decreto No. 131. Constitución Política de la República de Honduras. Diario Oficial La Gaceta No. 23,612. Tegucigalpa. 2 de enero de 1982. Retrieved from http://www. poderjudicial.gob.hn/CEDIJ/Leyes/Documents/Constituci\%C3\%B3n\%20de\%20la\%20Rep\%C3\%BAblica\%20 de\%20Honduras\%20\%28Actualizada\%202014\%29.pdf

W Decreto No. 286-2009. Ley para el establecimiento de una visión de país y la adopción de un plan de nación para Honduras. Diario Oficial La Gaceta No. 32,129. Tegucigalpa. Martes 2 de febrero de 2010.. Retrieved from http://www.andi.hn/wp-content/uploads/2012/11/ ley_para_el_establecimiento_de_una_vision_de_pais.pdf

- Economía y Educación. (1977). Carnoy, M. (Universidad de Chicago Press, Ed.). Chicago. Retrieved from http://externos.uma.es/cuadernos/pdfs/pdf279.pdf

Flores, Manuel. (2007). La Transición Demográfica en
Honduras. (Fondo de Población de las Naciones Unidas, Ed.). Tegucigalpa.

Flores Crespo, P. (2008). Análisis de política pública en educación: línea de investigación. Retrieved from http://www.uia.mx/web/files/inide5.pdf

Freitez, A. (18 de febrero a 26 noviembre). Transición Demográfica y Demandas de Educación Primaria y Media. Centro de Reflexión y Planificación Educativa. Educación para transformar el país, Venezuela. Retrieved from http://www.cerpe.org.ve/tl_files/Cerpe/contenido/documentos/Actualidad\%20Educativa/16\%20-\%20Demografia\%20y\%20Educacion\%20-\%20Freitez.pdf

Imprenta Nacional de Colombia. (2009). Metodología Proyecciones de Población y Estudios Demográfico. Retrieved from http://www.banrep.gov.co/sites/default/files/ paginas/Proyecciones_poblacion.pdf

Observatorio Económico y del Emprendimiento. (OEE). Retrieved from http://oee.iies-unah.org/index.php/demografico-social/tasa-gobal-de-fecundidad-tgf

INE. Honduras (2014). Estimaciones y Proyecciones de Población. Tegucigalpa.

Ruiz, D. (2007). La extraedad escolar: ¿Una anormalidad social? Textos Unversitarios. Venezuela: Publicaciones Vicerrectorado Académico. Retrieved from http://www. serbi.ula.ve/serbiula/librose/pva/Libros\%20de\%20PVA\%20 para\%20libro\%20digital/la\%20extraedad\%20escolar.pdf

W Santillana (Ed.). (1988, (C1983). Diccionario de las ciencias de la educación (Ed. rev. y actualizada en un vol). Madrid: Aula; Santillana.

Secretaría de Finanzas. (SEFIN). Presupuesto por finalidad. Enero 2007. Retrieved from http://www.sefin. gob.hn/data/2007/dgp/Presupuestos/presupuesto2006/ estadisticas/r_fpr_finfun_..pdf

- Secretaría de Finanzas. (SEFIN). Presupuesto por finalidad. Enero 2012. Retrieved from http://www.sefin. gob.hn/data/2011/DGP/PresupuestoAprobado2011/estadisticas/r_fpr_finfun.pdf

Secretaría de Finanzas. (SEFIN). Presupuesto aprobado por entidad. Ejercicio fiscal 2006. Enero 2007. Retrieved from http://www.sefin.gob.hn/data/2007/dgp/Presupuestos/presupuesto2006/estadisticas/r_fpr_finfun_.pdf

Secretaría de Finanzas. (SEFIN). Presupuesto aprobado por entidad. Ejercicio fiscal 2011. Enero 2012. Retrieved from http://www.sefin.gob.hn/data/2007/dgp/Presupuestos/presupuesto2006/estadisticas/r_fpr_finfun__.pdf 